\title{
Injury to Superior Vena Cava
}

National Cancer Institute

\section{Source}

National Cancer Institute. Injury to Superior Vena Cava. NCI Thesaurus. Code C78365.

Damage to the superior vena cava from traumatic or pathologic processes. 